\title{
BMJ Open Self-reported body silhouette trajectories across the lifespan and excessive daytime sleepiness in adulthood: a retrospective analysis. The Paris Prospective Study III
}

Quentin Lisan, ${ }^{1,2}$ Muriel Tafflet, ${ }^{1,2}$ Marie-Aline Charles, ${ }^{3}$ Frédérique Thomas, ${ }^{4}$ Pierre Boutouyrie, ${ }^{1,2,5}$ Catherine Guibout, ${ }^{1,2}$ José Haba-Rubio, ${ }^{6}$ Marie Cécile Périer, ${ }^{1,2}$ Bruno Pannier, ${ }^{4}$ Pedro Marques-Vidal, ${ }^{6,7}$ Xavier Jouven, ${ }^{1,2,8}$ Jean-Philippe Empana ${ }^{1,2}$

To cite: Lisan Q, Tafflet M, Charles M-A, et al. Selfreported body silhouette trajectories across the lifespan and excessive daytime sleepiness in adulthood: a retrospective analysis. The Paris Prospective Study III. BMJ Open 2018;8:e020851. doi:10.1136/ bmjopen-2017-020851

- Prepublication history and additional material for this paper are available online. To view these files, please visit the journal online (http://dx.doi. org/10.1136/bmjopen-2017020851).

Received 28 November 2017 Revised 2 February 2018 Accepted 6 March 2018
Check for updates

For numbered affiliations see end of article.

Correspondence to Dr Quentin Lisan; quentin.lisan@gmail.com

\section{ABSTRACT}

Objectives Excessive daytime sleepiness (EDS) is a common sleep complaint in the population and is increasingly recognised as deleterious for health. Simple and sensitive tools allowing identifying individuals at greater risk of EDS would be of public health importance. Hence, we determined trajectories of body silhouette from early childhood to adulthood and evaluated their association with EDS in adulthood.

Design A retrospective analysis in a prospective community-based study.

Participants 6820 men and women self-reported their silhouette at ages 8, 15, 25, 35 and 45 using the body silhouettes proposed by Stunkard et al. EDS was defined by an Epworth Sleepiness Scale score $\geq 11$.

Main outcome measure Presence of EDS in adulthood. Results The study population comprised 6820 participants (mean age 59.8 years, $61.1 \%$ men). Five distinct body silhouettes trajectories over the lifespan were identified: $31.9 \%$ 'lean stable', $11.1 \%$ 'lean increase', $16.1 \%$ 'lean-marked increase', $32.5 \%$ 'moderate stable' and $8.4 \%$ 'heavy stable'. Subjects with a 'heavy-stable' trajectory (OR 1.24, 95\% $\mathrm{Cl} 0.94$ to 1.62) and those with a 'Iean-marked increase' trajectory (OR 1.46, 95\% Cl 1.18 to 1.81) were more likely to have EDS when compared with the 'lean-stable' group after adjusting for confounding. Further adjustment for birth weight strengthened the magnitude of the ORs.

Conclusion Increasing body silhouette and to a lesser extent constantly high body silhouette trajectory from childhood to adulthood are associated with increased likelihood of EDS, independently of major confounding variables.

Trial registration number NCT00741728; Pre-results.

\section{INTRODUCTION}

Excessive daytime sleepiness (EDS) is highly common in the general population. Its prevalence has been reported up to $28 \%,{ }^{1-4}$ and is expected to rise. ${ }^{5}$ EDS has been associated

\section{Strengths and limitations of this study}

- Large sample size in a community-based study.

- We used a new, lifetime approach to estimate the evolution of body shape and weight over the lifespan, bringing information beyond one single measure of adiposity (such as body mass index or waist circumference).

- Retrospective recall of body silhouettes.

- No objective measures of sleep disorders (polysomnography).

with an increased risk of occupational injuries, ${ }^{6}$ and in addition with a twofold increased risk of cardiovascular morbidity and up to $70 \%$ increased risk of mortality. ${ }^{7}$ However, the determinants of EDS remain to be firmly established to consider interventions that may help preventing EDS. Given the epidemic of obesity and overweight worldwide, ${ }^{8}$ establishing a relationship between anthropometric markers and EDS would carry major public health implications. Associations between waist circumference or body mass index (BMI) and EDS have been reported in some, ${ }^{9-12}$ but not all studies. ${ }^{13} 14$ One possible explanation for these discrepancies is that most previous studies were cross-sectional so that they focused on one single measure of these anthropometrics markers. ${ }^{9-11} \mathrm{BMI}$, as well as waist circumference, change over the lifespan so that correlating changes in body weight with future EDS might bring more accurate predictive information. Unfortunately, studies with repeated measurements of BMI or waist circumference and with information on EDS are scarce. So far, 
only two studies, including one conducted specifically in women, evaluated the association between changes in BMI across two measures over time and the occurrence of EDS. ${ }^{12} 15$ These two studies considered BMI measures in adulthood only but not during infancy and adolescence, which represent critical periods regarding weight gain and weight attained in adulthood. ${ }^{16} 17$

Self-reported body silhouette has been shown to be a simple and reliable marker of body shape and weight. ${ }^{18-21}$ Recall of body silhouette at different ages correlates with objective measures of BMI at the corresponding ages. ${ }^{18} 19$ Hence, by combining recall of body silhouettes at different ages, it is possible to construct body silhouette trajectories over the life course. Accordingly, some recent studies have related self-reported body silhouette trajectories over the lifespan with different health-related outcomes including mortality, type 2 diabetes or cancer. ${ }^{20-23}$

In this study, we used a trajectory approach to investigate potential relationship between the trajectory of body silhouette from early childhood to adulthood and EDS in adulthood. In particular, we hypothesised that it is more the dynamic than the starting point value of the body silhouette that brings predictive information for EDS in adulthood.

\section{MATERIAL AND METHODS \\ Study population}

The design and main objectives of the Paris Prospective Study III have been published previously. ${ }^{24}$ It is an ongoing prospective observational cohort study in which 10157 men and women aged 50-75 years were recruited between June 2008 and June 2012. The standard health check-up included a complete clinical examination, coupled with standard biological tests after an overnight fast. A self-administered questionnaire provided information related to professional activity, lifestyle, personal and family medical history, current health status, and use of medications. Our study complies with the Declaration of Helsinki. All procedures performed in this study involving human participants were in accordance with the ethical standards of the institutional research committee and with the Declaration of Helsinki 1964 and its later amendments or comparable ethical standards. All the volunteers were recruited after signing an informed consent form.

\section{Sleep variables}

Excessive daytime sleepiness

Participants filled the Epworth Sleepiness Scale (ESS), which is a self-administered questionnaire containing eight questions evaluating the probability of dozing during daily activities, each question being rated from 0 (would never doze) to 3 (high chance of dozing), so that the ESS score ranges from 0 to 24. In accordance with the literature, we considered an ESS score of 11 and above to define EDS in main analysis. ${ }^{125}$ An ESS score $\geq 16$ was also considered to define severe EDS in sensitivity analysis (see end of "Statistical analyses" paragraph).
Sleep duration, snoring and sleep-related medications

Sleep pattern was obtained by questionnaire, such as usual sleeping and waking times. Information on the frequency (never, rarely, regularly or often) of loud snoring during night was obtained and we considered 'regularly or often' snorers versus others. Working schedules were recorded (day or night working). A medical doctor checked current medication during a face-to-face interview with the study participants. WHO Anatomical Therapeutic Chemical (ATC) classification was used to categorise medications; in particular, sleep-related medications (ie, that may impact on sleep) were identified by ATC code N05.

\section{Proxy for sleep-disordered breathing}

In the absence of objective sleep-disordered breathing (SDB) measures, we constructed a proxy for SDB. A participant was considered having a plausible SDB when he/she had loud snoring and a cluster of male sex and/or BMI $>30 \mathrm{~kg} / \mathrm{m}^{2}$ and/or age 55 or older and/or hypertension.

\section{Body silhouette assessment}

We used body silhouettes adapted from those proposed by Stunkard et al. ${ }^{26}$ At study recruitment, participants were asked to recall and choose one body silhouette among seven proposed for ages $8,15,25,35$ and 45 years. Body silhouettes were presented using children and adult silhouettes (figure 1).

\section{Covariates}

Depression score was assessed using the 13-item Questionnaire of Depression second version, Abridged (QD2A). ${ }^{27}$ Depressive status (yes/no) was defined by a QD2A score $\geq 7$. Prevalent cardiovascular disease, including history of stroke, myocardial infarction or angina pectoris, was self-reported. Diabetes was defined either by the intake of diabetes therapy or a fasting blood glucose $>7 \mathrm{mmol} / \mathrm{L}$. Blood pressure was measured after $10 \mathrm{~min}$ of rest in a subject in decubitus. Hypertensive status was defined either by the intake of antihypertensive treatment or a systolic blood pressure $\geq 140 \mathrm{~mm}$ $\mathrm{Hg}$ or a diastolic blood pressure $\geq 90 \mathrm{~mm} \mathrm{Hg}$. Habitual physical activity was assessed using the Baecke's score. ${ }^{28}$ A higher score corresponds to higher physical activity. Subjects were also asked to report if their birth weight was below $2.5 \mathrm{~kg}$, between 2.5 and $4 \mathrm{~kg}$ or above $4 \mathrm{~kg}$. Since collecting data about ethnicity is forbidden by law in France, ethnicity was assessed from the participant's and the parents' country of birth. ${ }^{29} \mathrm{We}$, therefore, considered Caucasian, African and non-Caucasian non-African.

\section{Statistical analyses}

To construct body silhouette trajectories, we selected subjects who reported their body silhouettes for at least three age periods among the five, including necessarily the earliest and the latest period. Since body silhouettes across the lifespan are longitudinal data, we used a groupbased trajectory modelling implemented by SAS Proc Traj. ${ }^{30}$ We identified five body silhouette trajectories, 


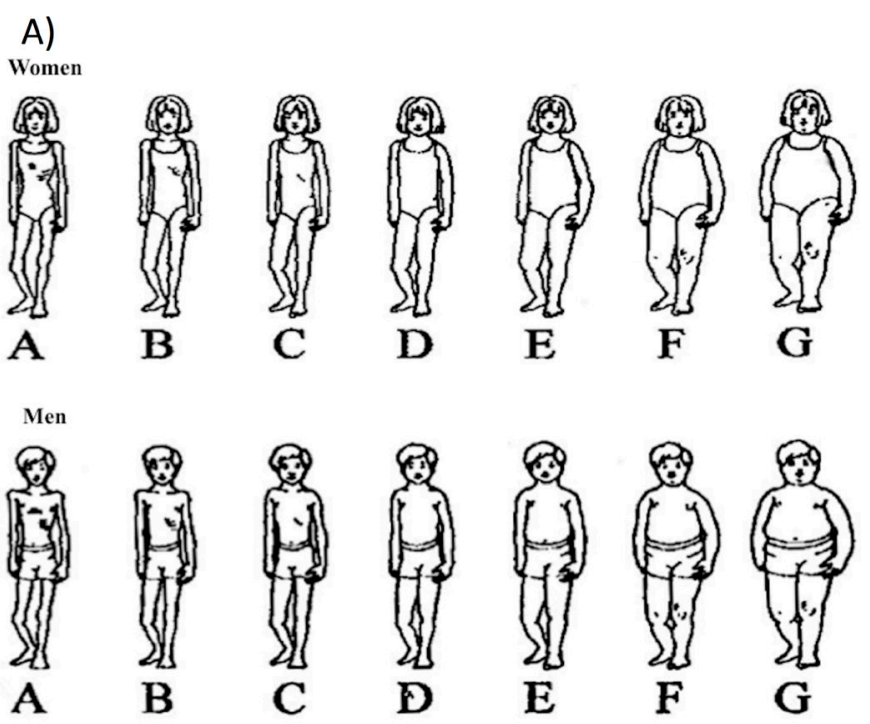

B)

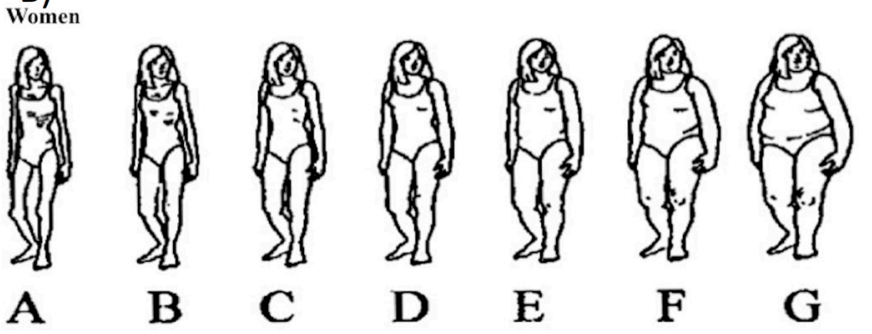

A

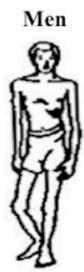

A

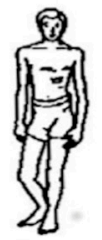

B

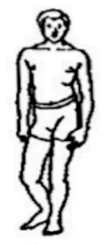

C

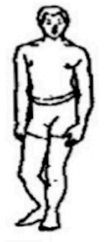

D $\mathbf{E}$

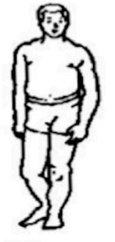

E

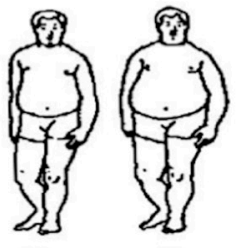

F $\quad \mathbf{G}$

Figure 1 Body silhouettes in $(A)$ children and (B) adults, adapted from Stunkard et al. ${ }^{26}$

based on the change in the Bayesian information criterion (figure 2). Then, we calculated the posterior probability for each participant to belong to each of the five trajectories and attributed the participant to a trajectory according to the highest probability. The average probabilities for each group were $86 \%, 89 \%, 79 \%, 89 \%$ and $91 \%$, indicating a good allocation of subjects among trajectories. Analysis was initially performed by sex, but trajectories were almost identical between men and women, and there was no significant interaction between sex and group-based trajectories ( $p$ value for interaction $=0.62$ ). Similarly, there was no significant interaction between body silhouette trajectories and age at inclusion $(\mathrm{p}=0.83)$. Hence, body silhouettes trajectories were described without stratification by gender or age.

The reliability of the obtained trajectories was further tested using the $\mathrm{kml}$ package of $\mathrm{R}$ software. This method is based on an implementation of the k-means clustering

algorithm adapted for longitudinal data. ${ }^{31}$ According to quality criteria (Calinski and Harabasz, Ray and Turi and Davies and Bouldin) ${ }^{31}$ the optimal number of trajectories was five. Using this approach, we found very similar body silhouette trajectories as the previous ones (online supplementary figure 1).

Multivariable logistic regression modelling was then used to quantify the relationship between body silhouette trajectory and EDS, estimating OR and 95\% CIs. To be more specific regarding the body silhouette trajectories assignment, we repeated the main analyses after considering only subjects with a posterior probability of belonging to one trajectory group $\geq 80 \%$.

To investigate the robustness of the results, we conducted several sensitivity analyses. To account to the fact that BMI at study recruitment may partly influence the body silhouette trajectory, we adjusted the previous multivariable model on BMI at study recruitment, keeping in mind that overadjustment may be present. Furthermore, to test whether early life factors such as birthweight impact future body shape, we additionally adjusted our analysis for birthweight categories (missing in 851 participants), as exact birth weight was not available. To minimise residual confounding by SDB, we then adjusted the model on loud snoring and then on the presence of a plausible SDB (missing in 538 subjects). To account for missing covariates, we reran the main analysis after using multiple imputations by chained equation. ${ }^{32}$ Finally, to test for an association with the severity of EDS, we considered moderate EDS (ESS between 11 and 14) and severe EDS (ESS $\geq 16$ ), and performed multinomial logistic regression.

All analyses were two sided and we considered a $\mathrm{p}<0.05$ as statistically significant. Statistical analyses were performed with R V.3.1.3 (www.r-project.org) and SAS V.9.3.

\section{RESULTS}

\section{Study population}

Body silhouette trajectory could be assessed in 7106 participants, of whom 6820 also had non-missing EDS. The study sample comprised $61.1 \%$ of men and the mean age was 59.8 years (SD 6.3 years). A total of 1154 participants $(16.9 \%)$ had EDS, $16.4 \%$ in women and $17.0 \%$ in men, respectively. The rates of EDS decreased with age, being $19.3 \%$ before $60,14 \%$ before $70 \%$ and $13.4 \%$ after 70 ( $\mathrm{p}$ for trend $<0.001$ ).

\section{Anthropometric markers at study recruitment and EDS}

As shown in online supplementary table 1, in multivariable analysis, EDS was associated with obesity (OR 1.53, 95\% CI 1.21 to 1.94), but not with overweight at study recruitment. Furthermore, in multivariable analysis, higher waist circumference was significantly associated with EDS (OR 1.01 per $1 \mathrm{~cm}$ increase, 95\% CI 1.00 to 1.02). However, the association did not reach statistical significance when considering clinical cut-off values 


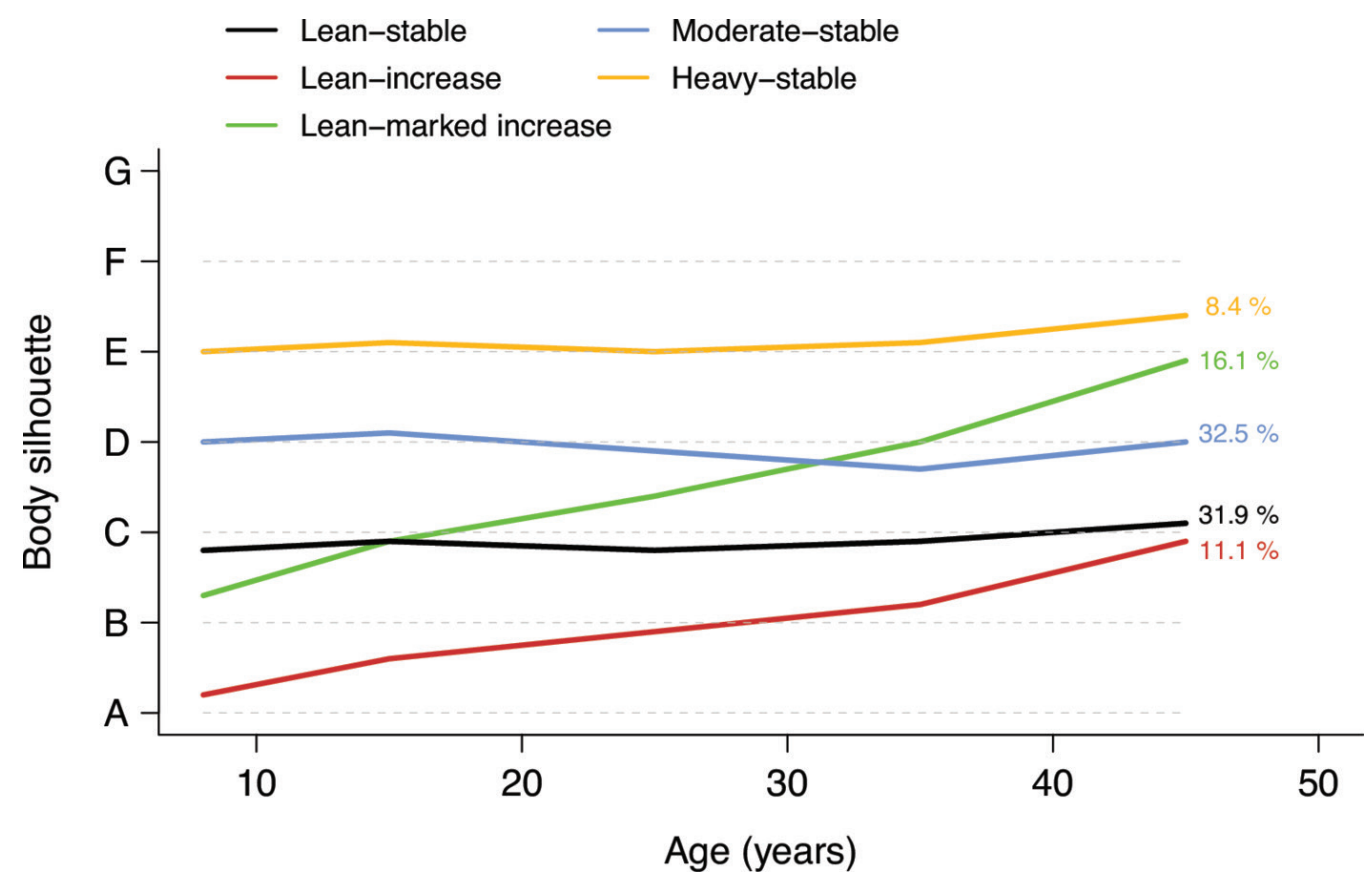

Figure 2 Trajectories of body silhouette. Note: percentages represent the proportion of the population according to each trajectory.

of $>88 \mathrm{~cm}$ ( 35 inches) for women and $>102 \mathrm{~cm}$ (40 inches) for men (OR $1.16,95 \%$ CI 0.96 to 1.40$)$.

\section{Age-specific silhouettes and EDS}

Table 1 summarises the associations between body silhouette at each age $(8,15,25,35$ and 45 years) and EDS, after adjusting for sex and age at inclusion. Only early and extreme body silhouettes were associated with EDS. Accordingly, we found strong and significant associations between EDS and the heaviest silhouette at age 8 (OR $2.59,95 \%$ CI 1.14 to 5.54$)$. There was also a moderate association between EDS and very lean body silhouette at age 8 (OR 1.30, 95\% CI 1.03 to 1.64 ).

\section{Body silhouette trajectories analysis \\ Main analyses}

From group-based trajectory modelling, five different trajectories were identified (figure 2): $31.9 \%$ were classified as 'lean stable', $11.1 \%$ as 'lean increase', $16.1 \%$ as 'lean-marked increase', $32.5 \%$ as 'moderate stable' and $8.4 \%$ as 'heavy stable'. Characteristics of participants according to body silhouette trajectories are presented in table 2. In particular, 'lean-marked increased' and 'heavy-stable' trajectories exhibited the highest rates of EDS, loud snoring, SDB (proxy) and depression. They also had the highest mean BMI values compared with the remaining trajectories. Noteworthy, the proportion of babies born above $4 \mathrm{~kg}$ increased across the five trajectories ( $\mathrm{p}$ for trend $<0.001$ ).

In multivariable logistic regression (table 3) and compared with the 'lean-stable' trajectory, two trajectories demonstrated an association with EDS: the 'heavy-stable' trajectory (OR 1.24, 95\% CI 0.94 to 1.62 ) and the 'leanmarked increase' trajectory (OR 1.46, 95\% CI 1.18 to 1.81 ).
In these analyses, subjects with depressive symptoms and short time sleepers $(<6$ hours vs $6-8$ hours) were two times more likely to have EDS whereas ageing was related to a $3 \%$ less likelihood of EDS per 1-year increase (table 3). When focusing on subjects with a posterior probability of belonging to a group trajectory $\geq 80 \%$, associations with 'heavy stable' (OR 1.52, 95\% CI 1.12 to 2.04 ) and 'leanmarked increase' trajectory (OR 1.77, 95\% CI 1.34 to 2.32) were even higher (online supplementary table 2 ).

\section{Sensitivity analyses}

The results of the sensitivity analyses are reported on online supplementary table 3. Overall, ORs were consistent with those from the main analysis after successively adjusting for BMI categories, loud snoring and a proxy for SDB, and after performing multiple imputations of missing covariates. Further adjustment for birthweight categories strengthened the magnitude of the ORs. Of note, in these analyses, neither BMI (except BMI $\geq 30 \mathrm{~kg} / \mathrm{m}^{2}$ ) nor birthweight categories were related to EDS, whereas loud snoring and SDB showed significant associations.

Of the 1154 participants with EDS, 953 had moderate EDS and 201 severe EDS. In multinomial logistic regression, the 'lean-marked increase' trajectory was associated with moderate EDS (OR 1.47, 95\% CI 1.17 to 1.85 ) and to a lesser extent with severe EDS (OR 1.41, 95\% CI 0.87 to 2.26). The 'heavy-stable' trajectory was associated with moderate EDS (OR 1.27, 95\% CI 0.95 to 1.70) but not with severe EDS (OR 1.06, 95\% CI 0.57 to 2.00 ).

\section{DISCUSSION}

In this large community-based study, five body silhouette trajectories were identified using self-reported body 
Table 1 Excessive daytime sleepiness (EDS) according to body silhouette in men and women at different age from childhood to adulthood

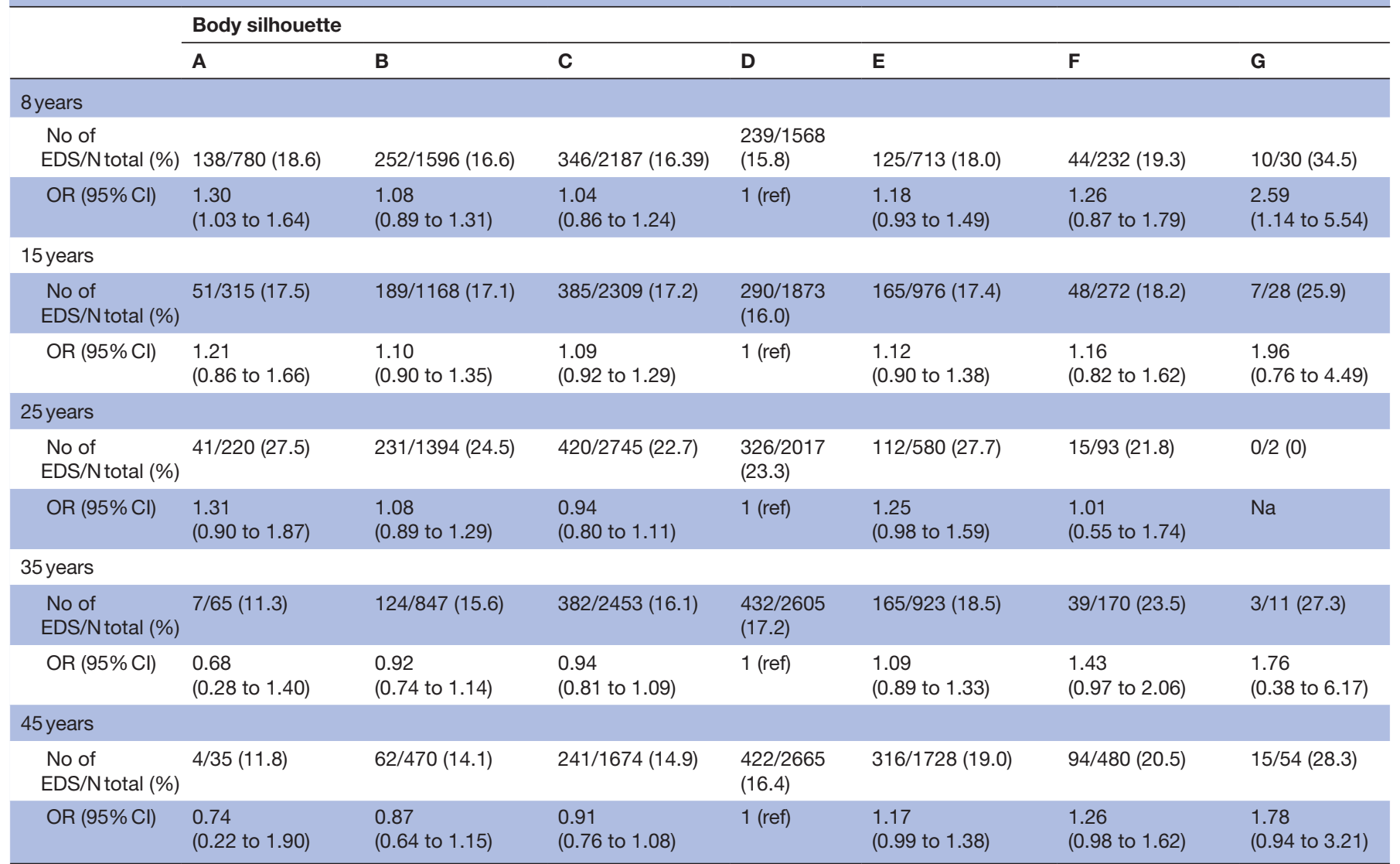

All ORs are adjusted for sex and age at inclusion.

$\mathrm{Na}$, not applicable due to zero observation; Ref, reference category.

silhouettes at different ages from childhood to adulthood. The trajectories were categorised as 'lean stable', 'lean increase', 'lean-marked increase', 'moderate stable' and 'heavy stable'. Compared with subjects with a 'leanstable' trajectory, those with a 'lean-marked increase' and 'heavy-stable' trajectories were the most likely to have EDS after accounting for several confounding factors.

In our study, separate analysis of body silhouette for each age indicates that extreme and early life body silhouettes were associated with EDS in adulthood. This was particularly true for the heavy body silhouette at 8 years of age, for which a 2.6-fold increased risk of EDS was observed. This may indicate that obesity during childhood is associated with sleep disorders in children, ${ }^{33}$ and plays a role in sleep disorders during adulthood. However, these figures should be interpreted with caution given the small size of this subgroup and the width of the $95 \%$ CI. On the other hand, this association is probably underestimated considering that the prevalence of obese children is currently growing and the fact that our population was born in the first part and in the middle of the 20th century. ${ }^{34}$

Data regarding the association of repeated assessment of adiposity with EDS are scarce. To date, only three studies have evaluated the association between changes in BMI and occurrence of EDS. ${ }^{12}{ }^{15}$ In 1395 middle-aged subjects from the Penn State Adult cohort, an association was found between weight gain 7.5 years apart and incident and persistent EDS, whereas weight loss was associated with EDS remission. ${ }^{12}$ The second study by Theorell-Haglöw et al also reported an association between weight gain 10 years apart and EDS in 4322 women. ${ }^{15}$ Lastly, in 1468 adults men and women from the Sleep Heart Health Study, every $10 \mathrm{~kg}$ gain measured 5 years apart was associated with a 0.55 point increase in the ESS score for women, whereas no association was reported in men. ${ }^{35}$ In these three studies, however, change in BMI was based on two repeated measures only; also, the three studies were conducted in adults only, so that trends in adiposity during infancy and adolescence were not available. Taken together, the results of these three studies may not give the full spectrum of change in BMI over the lifespan. In addition, only one study used a standardised and validated questionnaire to define EDS, ${ }^{35}$ while another one was conducted selectively in women. ${ }^{15}$

To the best of our knowledge, this is the first study associating body silhouette trajectories from childhood to adulthood with future EDS in adulthood. Two body silhouette trajectories were associated with EDS in adulthood. Our results on the 'lean-marked increase' trajectory are consistent with previous studies reporting that children 
Table 2 characteristics of participants according to body silhouette trajectories

\begin{tabular}{|c|c|c|c|c|c|c|}
\hline & \multicolumn{6}{|c|}{ Body silhouette trajectory } \\
\hline & $\begin{array}{l}\text { Lean stable } \\
\mathrm{n}=2269 \\
(31.9 \%)\end{array}$ & $\begin{array}{l}\text { Lean increase } \\
n=790(11.1 \%)\end{array}$ & $\begin{array}{l}\text { Lean-marked } \\
\text { increase } \\
n=1147(16.1 \%)\end{array}$ & $\begin{array}{l}\text { Moderate stable } \\
n=2306(32.5 \%)\end{array}$ & $\begin{array}{l}\text { Heavy stable } \\
n=594(8.4 \%)\end{array}$ & P values \\
\hline Male sex, n (\%) & $1414(62.3)$ & 570 (72.2) & $852(74.3)$ & 1249 (54.2) & $281(47.3)$ & $<0.001$ \\
\hline Age, mean $\pm S D$ & $59.6 \pm 6.03$ & $60.5 \pm 6.35$ & $59.3 \pm 6.24$ & $59.6 \pm 6.25$ & $58.9 \pm 5.99$ & $<0.001$ \\
\hline $\begin{array}{l}\text { Alcohol consumption, } \\
\mathrm{n}(\%)\end{array}$ & - & - & - & - & - & $<0.001$ \\
\hline Never & 229 (10.2) & $73(9.4)$ & $125(11.0)$ & $239(10.5)$ & $65(11.0)$ & - \\
\hline 1-2 drinks a day & 1724 (76.8) & $586(75.1)$ & $828(72.5)$ & $1772(77.5)$ & 477 (80.5) & - \\
\hline$\geq 3$ drinks a day & $292(13.0)$ & $121(15.5)$ & $188(16.5)$ & 275 (12.0) & $50(8.5)$ & - \\
\hline Education level, n (\%) & - & - & - & - & - & $<0.001$ \\
\hline No graduation & $56(2.5)$ & $30(3.9)$ & $68(6.0)$ & $66(2.9)$ & $21(3.6)$ & - \\
\hline Under HSD & 469 (20.9) & $181(23.2)$ & 307 (26.9) & $492(21.5)$ & $160(27.0)$ & - \\
\hline$\geq \mathrm{HSD}$ & 1715 (76.6) & $569(72.9)$ & $765(67.1)$ & $1732(75.6)$ & $411(69.4)$ & - \\
\hline Depression, n (\%) & $79(3.5)$ & $35(4.4)$ & $59(5.15)$ & $102(4.4)$ & $48(8.1)$ & $<0.001$ \\
\hline Living alone, n (\%) & $565(25.0)$ & $179(22.7)$ & $245(21.4)$ & $584(25.4)$ & $170(28.7)$ & 0.007 \\
\hline $\begin{array}{l}\text { Baecke's score, } \\
\text { mean } \pm S D\end{array}$ & $6.32 \pm 1.45$ & $6.21 \pm 1.43$ & $6.19 \pm 1.44$ & $6.29 \pm 1.43$ & $6.28 \pm 1.54$ & 0.13 \\
\hline Diabetes, n (\%) & $39(1.7)$ & $34(4.3)$ & $74(6.5)$ & 75 (3.3) & $22(3.7)$ & $<0.001$ \\
\hline Hypertension, n (\%) & $655(29.1)$ & $290(37.1)$ & $458(40.1)$ & 805 (35.2) & 218 (36.9) & $<0.001$ \\
\hline EDS, n (\%) & $336(15.4)$ & $123(16.4)$ & $231(21.1)$ & $351(15.7)$ & $113(19.6)$ & 0.001 \\
\hline Prevalent CVD, n (\%) & $30(1.3)$ & $20(2.5)$ & $29(2.5)$ & $50(2.2)$ & $10(1.7)$ & 0.07 \\
\hline $\mathrm{BMI}$, mean $\pm \mathrm{SD}$ & $23.7 \pm 2.99$ & $24.1 \pm 3.13$ & $26.8 \pm 3.51$ & $25.3+3.50$ & $27.9 \pm 4.49$ & $<0.001$ \\
\hline Birth weight, n (\%) & - & - & - & - & - & $<0.001$ \\
\hline$<2.5 \mathrm{~kg}$ & $104(5.4)$ & $58(9.3)$ & $64(6.8)$ & $79(4.0)$ & $21(4.2)$ & - \\
\hline $2.5-4 \mathrm{~kg}$ & $1683(86.8)$ & $524(84.0)$ & $791(84.3)$ & $1688(85.8)$ & $401(80.0)$ & - \\
\hline$>4 \mathrm{~kg}$ & $151(7.8)$ & $42(6.7)$ & $83(8.9)$ & $201(10.2)$ & $79(15.8)$ & - \\
\hline $\begin{array}{l}\text { Waist circumference } \\
(\mathrm{cm}), \text { mean } \pm S D\end{array}$ & $83.4 \pm 10.8$ & $85.7 \pm 10.3$ & $91.7 \pm 11.0$ & $85.9 \pm 11.8$ & $90.6 \pm 13.8$ & $<0.001$ \\
\hline $\begin{array}{l}\text { Sleep-related } \\
\text { medications, n (\%) }\end{array}$ & $79(4.1)$ & $32(4.8)$ & $53(5.3)$ & $90(4.5)$ & $26(4.8)$ & 0.67 \\
\hline Night working, n (\%) & $33(1.5)$ & $16(2.0)$ & $21(1.8)$ & $29(1.3)$ & $8(1.4)$ & 0.50 \\
\hline $\begin{array}{l}\text { Sleep duration (hours), } \\
\text { n (\%) }\end{array}$ & - & - & - & - & - & 0.097 \\
\hline 6-9hours/night & $1913(87.6)$ & $639(85.5)$ & $921(83.4)$ & $1911(86.8)$ & 476 (85.3) & - \\
\hline$<6$ hours/night & $244(11.2)$ & $97(13.0)$ & $167(15.1)$ & $262(11.9)$ & 77 (13.8) & - \\
\hline$\geq 9$ hours/night & $28(1.3)$ & $11(1.5)$ & $16(1.5)$ & $28(1.3)$ & $5(0.9)$ & - \\
\hline Loud snoring, n (\%) & $667(31.4)$ & $237(32.3)$ & $469(42.8)$ & $686(32.0)$ & $223(40.0)$ & $<0.001$ \\
\hline Proxy for SDB, n (\%) & $648(30.5)$ & $229(31.2)$ & $452(41.3)$ & $652(30.4)$ & $210(37.7)$ & $<0.001$ \\
\hline $\begin{array}{l}\text { Proxy for ethnicity, } \mathrm{n} \\
\text { (\%) }\end{array}$ & - & - & - & - & - & $<0.001$ \\
\hline Caucasian & 1966 (88.2) & 641 (83.2) & 922 (81.3) & $1989(87.5)$ & $509(87.4)$ & - \\
\hline African & $195(8.7)$ & 99 (12.9) & $176(15.5)$ & $221(9.7)$ & $57(9.8)$ & - \\
\hline $\begin{array}{l}\text { Non-Caucasian, non- } \\
\text { African }\end{array}$ & $69(3.1)$ & $30(3.9)$ & $36(3.2)$ & $64(2.8)$ & $16(2.8)$ & - \\
\hline
\end{tabular}

BMI, body mass index; CVD, cardiovascular disease; EDS, excessive daytime sleepiness; HSD, high school diploma; SDB, sleep-disordered breathing. 
Table 3 OR of body silhouettes trajectories for excessive daytime sleepiness

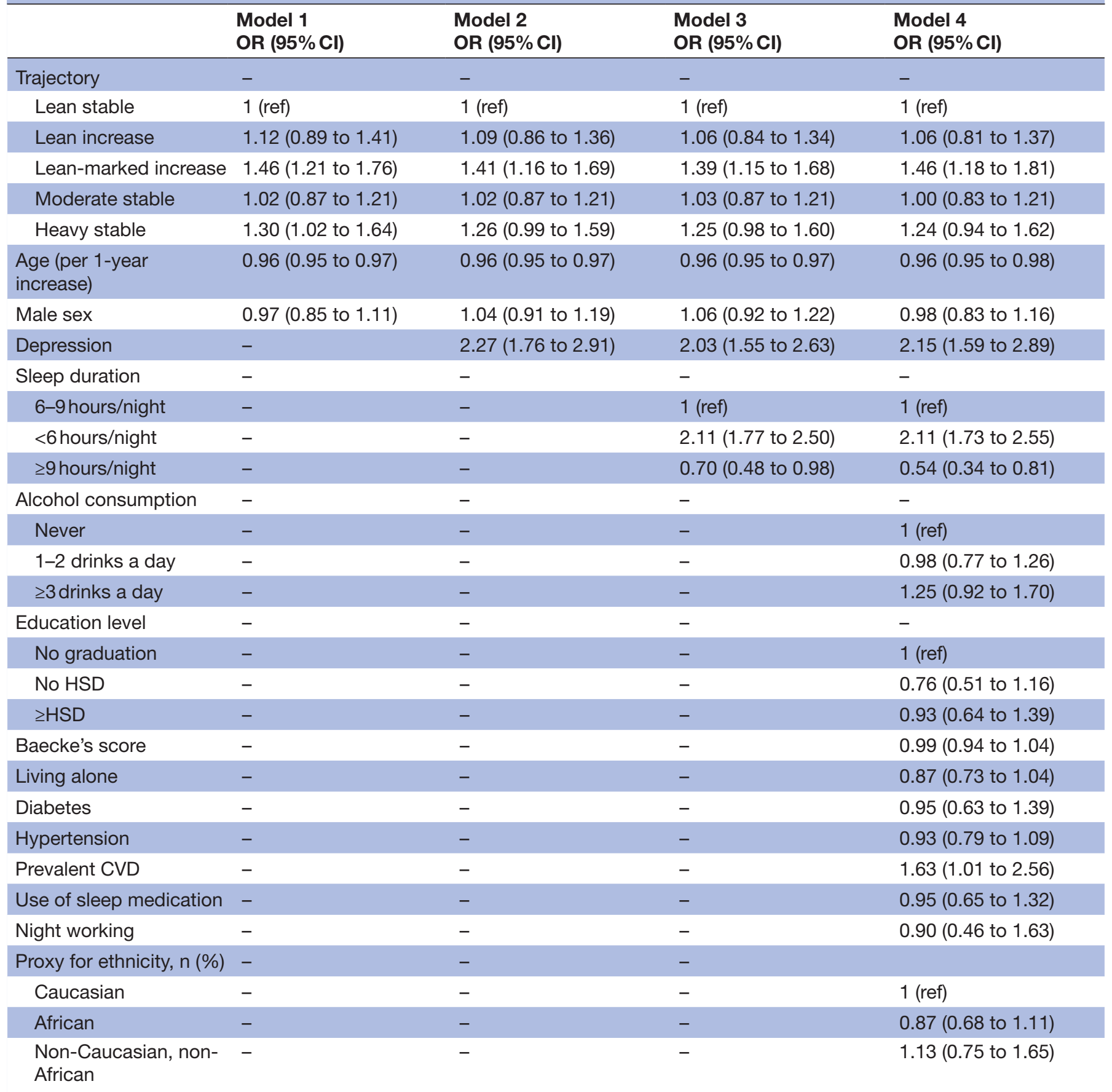

Model 1 is adjusted for age and sex, model 2: model 1+depression, model 3: model 2+sleep duration, model 4: model 3+all covariates listed in the table.

CVD, cardiovascular disease; HSD, high school diploma; Ref, reference category.

with a low birth weight and gaining weight rapidly until adolescence had a higher risk of medical conditions in adulthood, such as diabetes and coronary events. ${ }^{36} 37$ Hence, our study identified EDS as a novel detrimental consequence of such trajectory. The association of EDS with the 'heavy-stable' body silhouette trajectory, especially when investigating subjects with a posterior probability of belonging to one trajectory group $\geq 80 \%$, suggests that both the magnitude and the duration of excess weight may play a role in the development of EDS. This is consistent with previous studies showing that duration of excess weight and obesity was related to the risk of metabolic complications. ${ }^{38}$ Furthermore, the 'heavy-stable' trajectory may identify obesity with a strong genetic component that often develops early in life. ${ }^{39} 40$

In our study, the associations between 'lean-marked increase' and 'heavy-stable' body silhouette trajectories and EDS might reflect associations with either the last body silhouette measured at age 45 or the BMI at study recruitment. However, no association was found between 
the adult body silhouettes and EDS. Similarly, BMI at study recruitment did not impact the association between 'lean-marked increase' and 'heavy-stable' body silhouette trajectories and EDS. This indicates that the body silhouette lifetime approach provides information on the likelihood of EDS in adulthood beyond the one given by the last body silhouette or BMI.

Furthermore, the extent to which our study results reflect the effect of body silhouette trajectory per se or the effect of confounding factors associated with a pattern of body silhouette trajectory is a challenging issue. Psychological factors should be considered in this context. ${ }^{2}{ }^{13}$ However, despite the strong association between depressive symptoms and EDS, the relationship between body silhouette trajectory and EDS remained after adjusting for depressive symptoms. Also, the extent to which our study results reflect the effect of body silhouette trajectory on EDS or on related sleep disorders or sleep patterns that correlate with EDS deserve to be studied. In our study, associations between EDS and body silhouette trajectories were still present after adjustment for sleep duration and for a proxy for SDB, and despite the associations between EDS and these two sleep variables.

\section{Clinical implications}

Given the prevalence of EDS and its deleterious consequences on a wide spectrum of health-related outcomes, identifying modifiable determinants is of public health importance. Our study results suggest that body silhouette trajectory may bring information beyond BMI or waist circumference. Therefore, in addition to these two anthropometric measurements, body silhouette trajectories could be easily performed during an officebased consultation to identify subjects at increased risk of several medical conditions, including EDS. From our study results, it appears that such evaluation should start early in childhood. In addition to screening and intervening on depression, sleep duration and SDB, which have shown here and in other studies to be determinants of EDS, subjects constantly gaining weight may be proposed weight reduction programmes even in the absence of obesity. ${ }^{12}$

\section{Study limitations}

Despite the size of our study, the consideration of both men and women, and the use of a standardised tool to define subjective EDS, we acknowledge the following limitations. First, we relied on self-reported body silhouettes and not on objectively measured anthropometric markers. However, self-reported body silhouettes are well correlated with objective measures of BMI. ${ }^{18}{ }^{19}$ Second, body silhouettes at different age periods were not evaluated prospectively but retrospectively at study recruitment when participants were adult. However, recall of body silhouette at different ages correlates well with objective BMI measurements at the corresponding ages. ${ }^{18}$ Third, the last body silhouette refers to age 45 , while age at study entry ranged from 50 to 75 . However, body silhouette trajectories were not influenced by age at study entry in our study. Fourth, the modelling of trajectories being cohort dependent, our results may not be appropriate in other populations. Nevertheless, the trajectories found in our study are very similar to trajectories obtained in other studies. ${ }^{20}{ }^{23}$ Fifth, we had no objective polysomnographical records to estimate night sleep duration or the presence of a SDB, and instead used self-reported night sleep duration and a proxy for SDB.

In summary, in this community-based cohort, increasing and constantly high body silhouette trajectories from childhood to adulthood were associated with increased likelihood of EDS, independently of major confounding variables. This trajectory approach allowed us to identify new clusters of subjects at risk of EDS, independently of BMI, sleep duration and proxy for SDB.

\section{Author affiliations}

${ }^{1}$ Faculté de Médecine, Université Paris Descartes, Sorbonne Paris Cité, Paris, France ${ }^{2}$ Department of Epidemiology, INSERM, UMR-S970, Paris Cardiovascular Research Center, Paris, France

${ }^{3}$ INSERM, UMR1153, Center for Research in Epidemiology and Statistics Sorbonne Paris Cité (CRESS), Early ORigins of Child Health And Development Team (ORCHAD), Villejuif, France

${ }^{4}$ Preventive and Clinical Investigation Center, Paris, France

${ }^{5}$ Department of Pharmacology, AP-HP, Georges Pompidou European Hospital, Paris, France

${ }^{6}$ Center for Investigation and Research in Sleep, Lausanne University Hospital, Lausanne University, Lausanne, Switzerland

${ }^{7}$ Department of Medicine, Service of Internal Medicine, Lausanne University Hospital, Lausanne, Switzerland

${ }^{8}$ Cardiology Department, AP-HP, Georges Pompidou European Hospital, Paris, France

Contributors QL conducted statistical analyses, drafted the manuscript and performed the literature search. MT conducted statistical analyses, performed quality control of the data and reviewed the manuscript. M-AC reviewed the manuscript and revised it for important intellectual content, in particular for the Introduction and Discussion sections. FT participated in study design, acquisition of the data, supervised the field activities and reviewed the manuscript. PB participated in study design, acquisition of the data and reviewed the manuscript. CG participated in study design, acquisition of the data and its implementation, alongside with quality control and reviewed the manuscript. JH-R reviewed the manuscript and revised it for important intellectual content, in particular for the Discussion section. MCP performed quality control of the data and reviewed the manuscript. BP participated in study design, acquisition of the data, supervised the field activities and reviewed the manuscript. PM-V reviewed the manuscript and revised it for important intellectual content, in particular for the Discussion section. $\mathrm{XJ}$ participated in study design and reviewed the manuscript. J-PE participated in study design, acquisition of the data, supervised the field activities, participated in quality control, revised the manuscript for major intellectual content and gave final approval to submit the manuscript.

Funding The PPS3 Study was supported by grants from the National Research Agency (ANR), the Research Foundation for Hypertension (RFHTA), the Research Institute in Public Health (IRESP) and the Region lle de France (Domaine d'Intérêt Majeur).

Competing interests None declared.

Patient consent Obtained.

Ethics approval Ethics Committee of the Cochin Hospital (Paris, France).

Provenance and peer review Not commissioned; externally peer reviewed.

Data sharing statement No additional data are available.

Open Access This is an Open Access article distributed in accordance with the Creative Commons Attribution Non Commercial (CC BY-NC 4.0) license, which permits others to distribute, remix, adapt, build upon this work non-commercially, and license their derivative works on different terms, provided the original work is 
properly cited and the use is non-commercial. See: http://creativecommons.org/ licenses/by-nc/4.0/

(C) Article author(s) (or their employer(s) unless otherwise stated in the text of the article) 2018. All rights reserved. No commercial use is permitted unless otherwise expressly granted.

\section{REFERENCES}

1. Johns M, Hocking B. Daytime sleepiness and sleep habits of Australian workers. Sleep 1997;20:844-7.

2. Whitney CW, Enright PL, Newman AB, et al. Correlates of daytime sleepiness in 4578 elderly persons: the Cardiovascular Health Study. Sleep 1998;21:27-36.

3. Walsleben JA, Kapur VK, Newman AB, et al. Sleep and reported daytime sleepiness in normal subjects: the Sleep Heart Health Study. Sleep 2004;27:293-8.

4. Boden-Albala B, Roberts ET, Bazil C, et al. Daytime sleepiness and risk of stroke and vascular disease: findings from the Northern Manhattan Study (NOMAS). Circ Cardiovasc Qual Outcomes 2012;5:500-7.

5. Ford ES, Cunningham TJ, Giles WH, et al. Trends in insomnia and excessive daytime sleepiness among U.S. adults from 2002 to 2012. Sleep Med 2015;16:372-8.

6. Melamed S, Oksenberg A. Excessive daytime sleepiness and risk of occupational injuries in non-shift daytime workers. Sleep 2002;25:315-22.

7. Empana JP, Dauvilliers Y, Dartigues JF, et al. Excessive daytime sleepiness is an independent risk indicator for cardiovascular mortality in community-dwelling elderly: the three city study. Stroke 2009;40:1219-24.

8. Baskin ML, Ard J, Franklin F, et al. Prevalence of obesity in the United States. Obes Rev 2005;6:5-7.

9. Hayley AC, Williams LJ, Kennedy GA, et al. Excessive daytime sleepiness and body composition: a population-based study of adults. PLoS One 2014;9:e112238.

10. Vgontzas AN, Bixler EO, Tan TL, et al. Obesity without sleep apnea is associated with daytime sleepiness. Arch Intern Med 1998;158:1333-7.

11. Bixler EO, Vgontzas AN, Lin HM, et al. Excessive daytime sleepiness in a general population sample: the role of sleep apnea, age, obesity, diabetes, and depression. J Clin Endocrinol Metab 2005;90:4510-5.

12. Fernandez-Mendoza J, Vgontzas AN, Kritikou I, et al. Natural history of excessive daytime sleepiness: role of obesity, weight loss, depression, and sleep propensity. Sleep 2015;38:351-60.

13. Tsuno N, Jaussent I, Dauvilliers $Y$, et al. Determinants of excessive daytime sleepiness in a French community-dwelling elderly population. J Sleep Res 2007;16:364-71.

14. Hayley AC, Williams LJ, Kennedy GA, et al. Excessive daytime sleepiness and metabolic syndrome: a cross-sectional study. Metabolism 2015;64:244-52.

15. Theorell-Haglöw J, Åkerstedt T, Schwarz J, et al. Predictors for Development of Excessive Daytime Sleepiness in Women: A Population-Based 10-Year Follow-Up. Sleep 2015;38:1995-2003.

16. Stettler N, Zemel BS, Kumanyika S, et al. Infant weight gain and childhood overweight status in a multicenter, cohort study. Pediatrics 2002;109:194-9.

17. Must A, Strauss RS. Risks and consequences of childhood and adolescent obesity. Int J Obes Relat Metab Disord 1999;23(Suppl 2):S2-11.

18. Must A, Willett WC, Dietz WH. Remote recall of childhood height, weight, and body build by elderly subjects. Am J Epidemiol 1993;138:56-64.
19. Tehard B, van Liere MJ, Com Nougué C, et al. Anthropometric measurements and body silhouette of women: validity and perception. J Am Diet Assoc 2002;102:1779-84.

20. Song M, Hu FB, Wu K, et al. Trajectory of body shape in early and middle life and all cause and cause specific mortality: results from two prospective US cohort studies. BMJ 2016;353:i2195.

21. Song M, Willett WC, Hu FB, Fb H, et al. Trajectory of body shape across the lifespan and cancer risk. Int J Cancer 2016;138:2383-95.

22. Fagherazzi G, Vilier A, Affret A, et al. The association of body shape trajectories over the life course with type 2 diabetes risk in adulthood: a group-based modeling approach. Ann Epidemiol 2015;25:785-7.

23. Fagherazzi G, Guillas G, Boutron-Ruault MC, et al. Body shape throughout life and the risk for breast cancer at adulthood in the French E3N cohort. Eur J Cancer Prev 2013;22:29-37.

24. Empana JP, Bean K, Guibout C, et al. Paris Prospective Study III: a study of novel heart rate parameters, baroreflex sensitivity and risk of sudden death. Eur J Epidemiol 2011;26:887-92.

25. Johns MW. A new method for measuring daytime sleepiness: the Epworth sleepiness scale. Sleep 1991;14:540-5.

26. Stunkard AJ, Sørensen T, Schulsinger F. Use of the Danish Adoption Register for the study of obesity and thinness. Res Publ Assoc Res Nerv Ment Dis 1983;60:115-20.

27. Pichot P. A self-report inventory on depressive symptomatology (QD2) and its abridged form (QD2). Assessment of depression: Springer, 1986:108-22.

28. Baecke JA, Burema J, Frijters JE. A short questionnaire for the measurement of habitual physical activity in epidemiological studies. Am J Clin Nutr 1982;36:936-42.

29. Thomas F, Pannier B, Safar ME. Impact of country of birth on arterial function in subjects living in France. J Am Soc Hypertens 2012;6:405-13

30. Jones BL, Nagin DS. Advances in group-based trajectory modeling and an SAS procedure for estimating them. Sociol Methods Res 2007;35:542-71.

31. Genolini C, Falissard B. KmL: a package to cluster longitudinal data. Comput Methods Programs Biomed 2011;104:e112-e121.

32. Buuren Svan, Groothuis-Oudshoorn K. mice: multivariate imputation by chained equations in R. J Stat Softw 2011;45:1-67.

33. Miller AL, Lumeng JC, LeBourgeois MK. Sleep patterns and obesity in childhood. Curr Opin Endocrinol Diabetes Obes 2015;22:41-7.

34. Lee JM, Pilli S, Gebremariam A, et al. Getting heavier, younger: trajectories of obesity over the life course. Int $J$ Obes 2010;34:614-23.

35. $\mathrm{Ng} \mathrm{WL}$, Orellana L, Shaw JE, et al. The relationship between weight change and daytime sleepiness: the Sleep Heart Health Study. Sleep Med 2017;36:109-18.

36. Barker DJ, Osmond C, Forsén TJ, et al. Trajectories of growth among children who have coronary events as adults. $N$ Engl $\mathrm{J}$ Med 2005;353:1802-9.

37. Forsén T, Eriksson J, Tuomilehto J, et al. The fetal and childhood growth of persons who develop type 2 diabetes. Ann Intern Med 2000;133:176-82.

38. Power $\mathrm{C}$, Thomas $\mathrm{C}$. Changes in $\mathrm{BMI}$, duration of overweight and obesity, and glucose metabolism: 45 years of follow-up of a birth cohort. Diabetes Care 2011;34:1986-91.

39. Elks CE, Loos RJ, Sharp SJ, et al. Genetic markers of adult obesity risk are associated with greater early infancy weight gain and growth PLoS Med 2010;7:e1000284.

40. Elks CE, Heude B, de Zegher F, et al. Associations between genetic obesity susceptibility and early postnatal fat and lean mass: an individual participant meta-analysis. JAMA Pediatr 2014;168:1122-30. 